University of New Hampshire

University of New Hampshire Scholars' Repository

Space Science Center

Institute for the Study of Earth, Oceans, and

Space (EOS)

2000

\title{
Detection of 6 November 1997 ground level event by Milagrito
}

\author{
A Falcone \\ University of New Hampshire - Main Campus \\ R Atkins \\ University of Utah \\ W Benbow \\ University of California - Santa Cruz \\ D Berley \\ University of Maryland - College Park \\ M L. Chen \\ University of Maryland - College Park
}

See next page for additional authors

Follow this and additional works at: https://scholars.unh.edu/ssc

Part of the Astrophysics and Astronomy Commons

\section{Recommended Citation}

Detection of 6 November 1997 ground level event by Milagrito Falcone for Milagro collaboration, A. D., AIP Conference Proceedings, 510, 574-578 (2000), DOI:http://dx.doi.org/10.1063/1.1303268

This Conference Proceeding is brought to you for free and open access by the Institute for the Study of Earth, Oceans, and Space (EOS) at University of New Hampshire Scholars' Repository. It has been accepted for inclusion in Space Science Center by an authorized administrator of University of New Hampshire Scholars' Repository. For more information, please contact Scholarly.Communication@unh.edu. 


\section{Authors}

A Falcone, R Atkins, W Benbow, D Berley, M L. Chen, D G. Coyne, B L. Dingus, D E. Dorfan, R W. Ellsworth, D Evans, L Fleysher, R Fleysher, G Gisler, J A. Goodman, T J. Haines, C M. Hoffman, S Hugenberger, L A.

Kelley, I Leonor, John R. Macri, Mark L. McConnell, J F. McCullough, J E. McEnery, R S. Miller, A I. Mincer, M F. Morales, P Nemethy, James M. Ryan, B Shen, A Shoup, G Sinnis, A J. Smith, G W. Sullivan, O T. Tumer, K Wang, M O. Wascko, S Westerhoff, D A. Williams, T Yang, and G B. Yodh 


\section{AIP | Proceedings}

\section{Detection of 6 November 1997 ground level event by Milagrito}

A. D. Falcone for Milagro collaboration

Citation: AIP Conference Proceedings 510, 574 (2000); doi: 10.1063/1.1303268

View online: http://dx.doi.org/10.1063/1.1303268

View Table of Contents: http://scitation.aip.org/content/aip/proceeding/aipcp/510?ver=pdfcov

Published by the AIP Publishing

\section{Articles you may be interested in}

Pulse Shape Discrimination on the INTEGRAL Imaging Spectrometer

AIP Conf. Proc. 510, 712 (2000); 10.1063/1.1307032

Position sensitive germanium detectors for the Advanced Compton Telescope

AIP Conf. Proc. 510, 794 (2000); 10.1063/1.1303307

Milagro: A TeV gamma-ray monitor of the Northern Hemisphere Sky

AIP Conf. Proc. 510, 642 (2000); 10.1063/1.1303280

A "Snapshot" survey of the gamma-ray sky at $\mathrm{GeV}$ energies

AIP Conf. Proc. 510, 611 (2000); 10.1063/1.1303275

A ground based installation for the detection of gamma rays with energy $50-2000 \mathrm{GeV}$

AIP Conf. Proc. 304, 711 (1994); 10.1063/1.45677 


\title{
Detection of 6 November 1997 Ground Level Event by Milagrito
}

\author{
A.D. Falcone ${ }^{1}$, for Milagro collaboration \\ ${ }^{1}$ Space Science Center, University of New Hampshire, Durham, NH 03824 USA
}

\begin{abstract}
Solar Energetic Particles (SEPs) with energies exceeding $10 \mathrm{GeV}$ associated with the 6 November 1997 solar flare/CME (coronal mass ejection) have been detected with Milagrito, a prototype of the Milagro Gamma Ray Observatory. While SEP acceleration beyond $1 \mathrm{GeV}$ is well established, few data exist for protons or ions beyond $10 \mathrm{GeV}$. The Milagro observatory, a ground based water Cherenkov detector designed for observing very high energy gamma ray sources, can also be used to study the Sun. Milagrito, which operated for approximately one year in 1997/98, was sensitive to solar proton and neutron fluxes above $\sim 4 \mathrm{GeV}$. In its scaler mode, Milagrito registered a rate increase coincident with the 6 November 1997 ground level event observed by Climax and other neutron monitors. A preliminary analysis suggests the presence of $>10 \mathrm{GeV}$ particles.
\end{abstract}

\section{INTRODUCTION}

Particle acceleration beyond $1 \mathrm{GeV}$ due to solar processes is well established (1), but its intensity and energy still amazes researchers. However, few data exist demonstrating acceleration of protons or ions beyond $10 \mathrm{GeV}(2,3)$. The energy upper limit of solar particle acceleration is unknown but is an important parameter, since it relates not only to the nature of the acceleration process, itself not ascertained, but also to the environment at or near the Sun where the acceleration takes place. The Milagro instrument, a water Cherenkov detector near Los Alamos, NM, is at $2650 \mathrm{~m}$ elevation with a geomagnetic vertical cutoff rigidity of $\sim 4 \mathrm{GV}$. It is sensitive to hadronic cosmic rays from approximately $5 \mathrm{GeV}$ to beyond $1 \mathrm{TeV}$. These primary particles are detected via Cherenkov light, produced by secondary shower particles, as they traverse a large $(80 \times 60 \times 8 \mathrm{~m})$ water-filled pond containing 723 photomultiplier tubes (228 PMTs for the prototype, Milagrito). This energy range overlaps that of neutron monitors (in the region $<10 \mathrm{GeV}$ ) such that Milagro complements the worldwide network of these instruments. These ground-based instruments, in turn, complement spacecraft cosmic ray measurements at lower energies. This suite of instruments may then be capable of measuring the full energy range of solar hadronic

CP510, The Fifth Compton Symposium, edited by M. L. McConnell and J. M. Ryan (c) 2000 American Institute of Physics 1-56396-932-7/00/ $\$ 17.00$ 
cosmic rays, with the goal of establishing a fundamental upper limit to the efficiency of the particle acceleration by the Sun.

Milagro's baseline mode (air shower telescope mode) of operation measures extensive air showers above $300 \mathrm{GeV}$ from either hadrons or gamma rays. A description of Milagro's capabilities as a VHE gamma ray observatory is available elsewhere (4). Milagro measures not only the rate of these events but also the incident direction of each event, thereby localizing sources. While performing these measurements, the instrument records the rate of photomultiplier hits (the scaler mode), with an intrinsic energy threshold of about $4 \mathrm{GeV}$ for the progenitor cosmic ray to produce at least one hit. The scaler mode provides data that are similar to those of a neutron monitor, while the telescope mode can significantly reduce background by pointing. With a proposed fast data acquisition system (DAQ) and modified algorithms for determining incident directions of muons, the energy threshold of Milagro's telescope mode will be reduced to $\sim 4 \mathrm{GeV}$ by detecting the $(\sim 300 \mathrm{kHz})$ single muons and mini muon showers. For now, this low energy threshold can only be achieved by using Milagro in the scaler mode, which is not capable of localizing sources. A description of the Milagro solar telescope mode can be found in another publication (5).

\section{SOLAR MILAGRO/MILAGRITO SCALER MODE}

In the scaler mode, a substantial portion of the rate recorded by Milagro (and Milagrito) is due to muons, and an integral measurement above threshold is performed. These data provide an excellent high energy complement to the network of neutron monitors, which has been, and continues to be, a major contributor to our understanding of solar energetic particle acceleration and cosmic rays. With Monte Carlo calculations, we estimated the effective areas of Milagrito to protons incident on the atmosphere isotropically, at zenith angles ranging from $0^{\circ}-60^{\circ}$ (Figure 1). The effective area curves for Milagro, which have been plotted for the sake of comparison, are for beamed protons. At $10 \mathrm{GeV}$, Milagro's scaler mode is at least an order of magnitude greater than the effective area of a sea level neutron monitor, with the effective area rising rapidly with energy, while Milagrito had approximately 4 times the effective area of a neutron monitor at $10 \mathrm{GeV}$. Pressure, temperature, and other diurnal corrections must be applied to the ground level scaler rate (6). We have begun to determine these correction factors for Milagro/Milagrito, and we find them to be reasonably consistent with past work with muon telescopes (7). However, these corrections are less important for transient (i.e. solar) events that rise above background quickly and have short durations. 


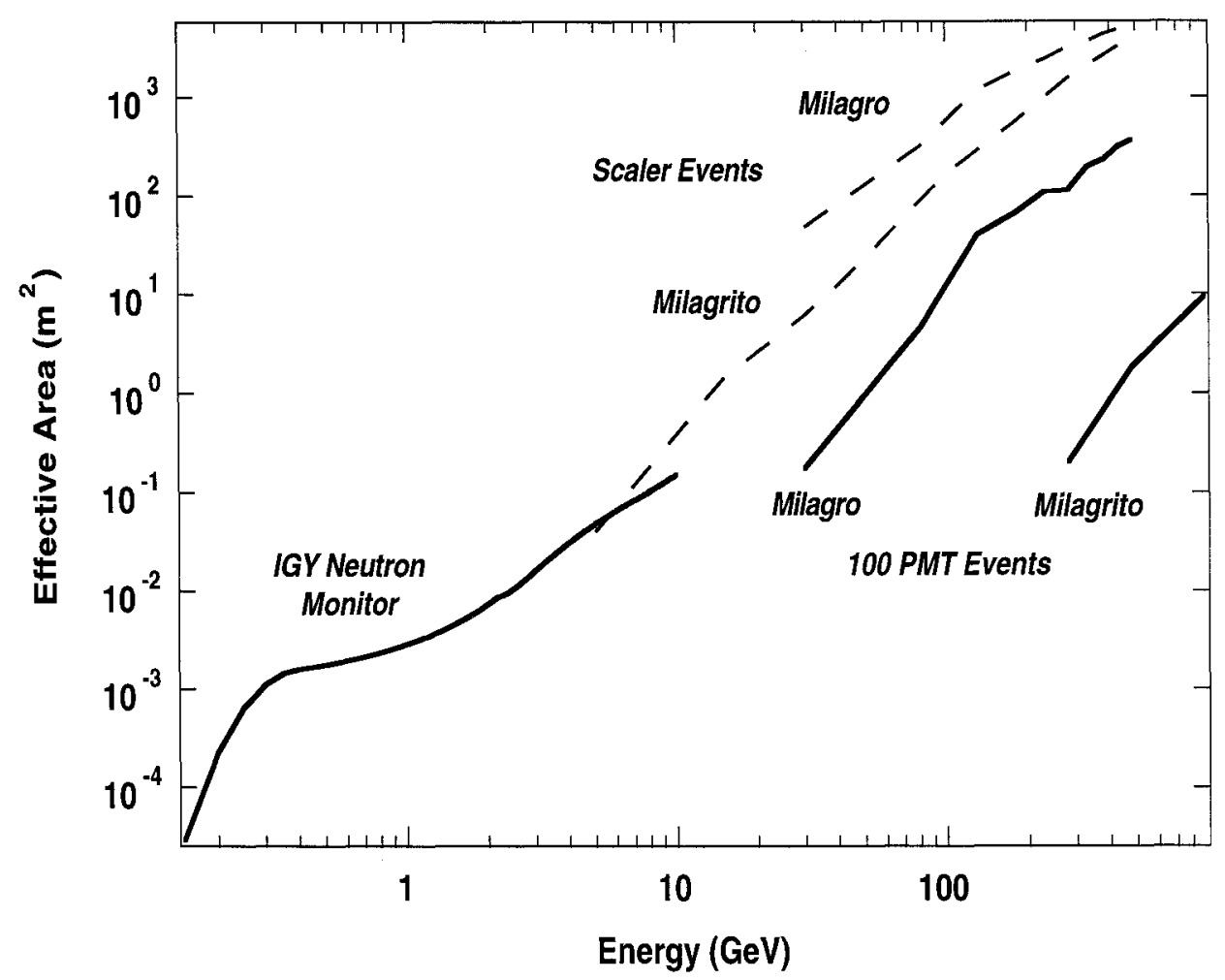

FIGURE 1. Preliminary effective area curves for Milagro and Milagrito, with a sea-level IGY neutron monitor for comparison. (The Milagro shower trigger is no longer set at 100 PMTs, but Milagrito shower data was recorded with a 100 PMT threshold. )

\section{NOVEMBER 1997 GROUND LEVEL EVENT}

On 6 November 1997 at approximately 12:00 UT, an X-class flare with an associated coronal mass ejection occurred on the Sun. This produced a nearly isotropic (8) ground level event registered by many neutron monitors. A preliminary analysis of neutron monitor data for this proton event yields a spectral index of 5.5 at event maximum in the $1-5 \mathrm{GV}$ rigidity range, assuming a power law rigidity spectrum for protons (9). Climax, located in nearby central Colorado, is the closest of these neutron monitors to Milagro/Milagrito. Milagrito, a prototype version of Milagro with less effective area, registered a scaler rate increase coincident, within error, with that measured by Climax (Figure 2). If one accounts for meteorological fluctuations, the event duration and time of maximum intensity, as seen by Milagrito, are also consistent with that of Climax. 


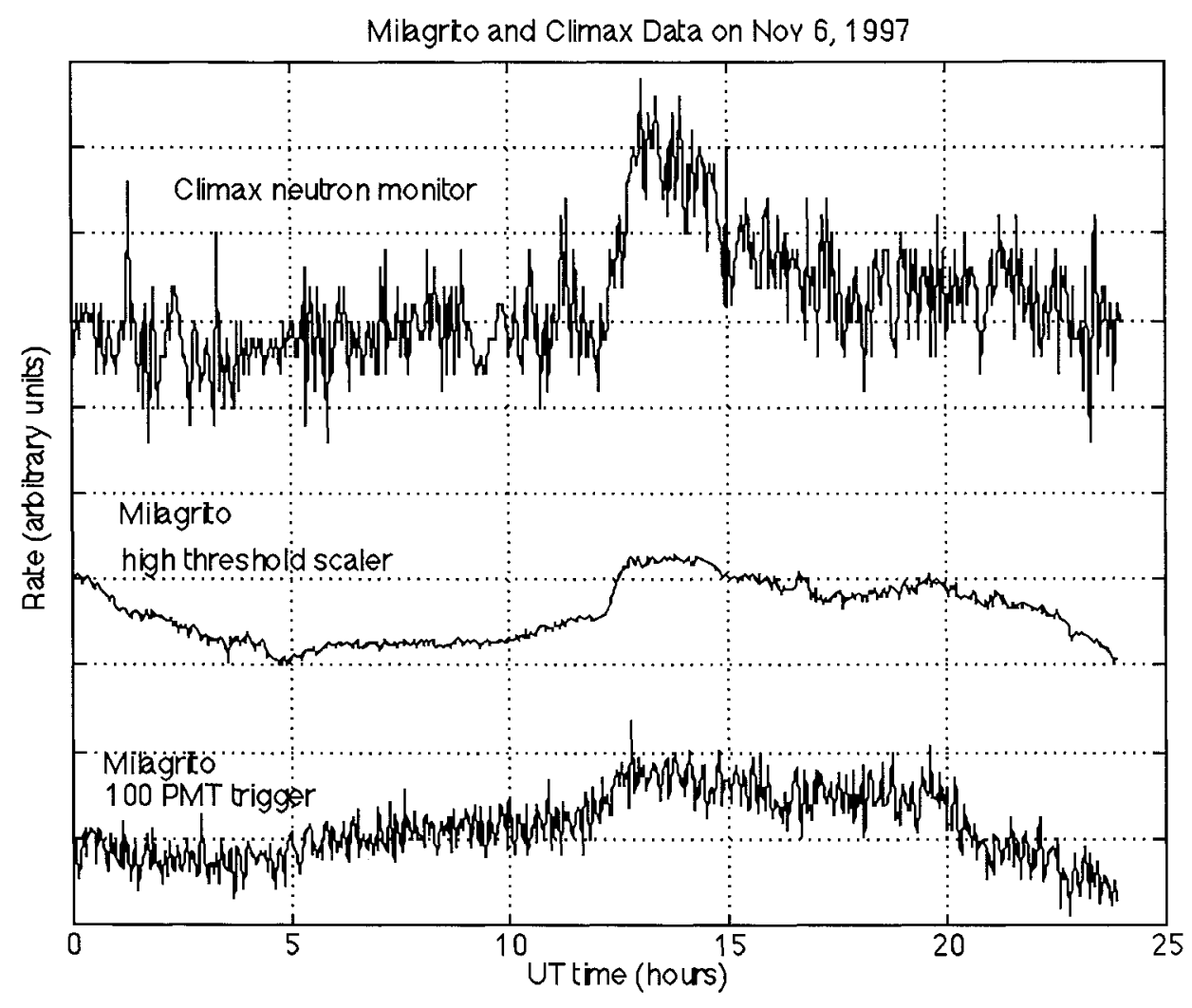

FIGURE 2. Milagrito registered a rate increase coincident with that of Climax during the GLE of Nov. 6, 1997. The $y$-axis units have been scaled and shifted for each plot to make comparison easier. (Climax data courtesy of C. Lopate, Univ. of Chicago)

The high threshold scaler rate increase can be used to derive characteristics of the primary proton spectrum. This is done by folding a trial power law spectrum of protons through the effective area of the detector. The parameters of the trial spectra are then varied until a good fit to the measured rate increase is achieved. When compared to the neutron monitor network's spectrum for protons $<4 \mathrm{GeV}$, the preliminary results of this analysis indicate the presence of protons in excess of 10 $\mathrm{GeV}$. Further work is necessary to better determine the spectrum at these energies.

The 100 PMT shower trigger rate also experienced an increase, although the significance is not as great as that in scaler mode. It is not yet clear which of several possible mechanisms initiated the signal in the 100 PMT shower trigger, so the detector's sensitivity to various mechanisms is being investigated. This increase could have been caused by high energy primaries (> $100 \mathrm{GeV}$, see Figure 1) and/or 
secondary muons arriving from a nearly horizontal direction. If horizontal secondary muons contributed to this signal, they would have been the result of high energy proton primaries, but the effective area of the detector would be significantly different from that assumed here and cannot be used to constrain the spectrum without more extensive Monte Carlo calculations. Future work will address this issue by considering events caused by horizontally incident secondary muons and recalculating the spectrum.

\section{ACKNOWLEDGMENTS}

This work is supported in part by the National Science Foundation, U.S. Department of Energy Office of High Energy Physics, U.S. Department of Energy Office of Nuclear Physics, Los Alamos National Laboratory, University of California, Institute of Geophysics and Planetary Physics, the Research Corporation, and the California Space Institute.

\section{REFERENCES}

1. Parker, E.N., Physical Review 107, 830 (1957).

2. Chiba, N., et al., Astroparticle Physics 1(1), 27-32 (1992).

3. Lovell, J.L., Duldig, M.L., Humble, J.E., Journal of Geophysical Research 103(A10), 23733 (1998).

4. McCullough, J.F., et al., "Status of the Milagro Gamma Ray Observatory," Proc.26 $6^{\text {th }}$ Int. Cosmic Ray Conf, 1999.

5. Falcone, A.D., et al., Astroparticle Physics 11(1-2), 283-285 (1999).

6. Hayakawa, S., Cosmic Ray Physics, New York: John Wiley and Sons, 1969.

7. Fowler, G.N., Wolfendale, A.W., S.Flügge, eds., Cosmic Rays I, 1961.

8. Duldig, M.L. \& Humble, J.E., "Preliminary Analysis of the 6 November 1997 Ground Level Enhancement," Proc.26 $6^{\text {th }}$ Int. Cosmic Ray Conf, 1999, Vol. 6, pp. 403-406.

9. Smart, D.F \& Shea, M.A., "Preliminary Analysis of GLE of 6 November 1997A," Proc. Spring American Geophysical Union Meeting, 1998. 